Check for updates

Cite this: RSC Adv., 2017, 7, 48263

Received 21st August 2017 Accepted 8th October 2017

DOI: $10.1039 / \mathrm{c} 7 \mathrm{ra09252k}$

rsc.li/rsc-advances

\section{Differentiating the impact of nitrogen chemical states on optical properties of nitrogen-doped graphene quantum dots $\dagger$}

\begin{abstract}
Timothy Pillar-Little (D) and Doo Young Kim (D) *
The optical properties of top-down synthesized oxidized graphene quantum dots (ox-GQDs) and nitrogenincorporating graphene quantum dots (N-GQDs) along a range of hydrothermal treatment temperatures were observed. By controlling the hydrothermal treatment temperature, different chemical states of nitrogen atoms were incorporated into GQDs. Below $150{ }^{\circ} \mathrm{C}$, edge-terminating amines and amides dominated the nitrogen content of $\mathrm{N}-\mathrm{GQDs}$. Above $150{ }^{\circ} \mathrm{C}$, nitrogen was primarily present in the forms of pyridinic, pyrrolic and quaternary N. In addition to the absorbance and emission profiles of ox-GQDs and $\mathrm{N}$-GQDs, pH-dependent emission spectra were collected to probe chemical states of nitrogen atoms and investigate the relationship between nitrogen location and photoluminescence.
\end{abstract}

Graphene quantum dots (GQDs) are the newest member in the functional carbon nanomaterials family. The unique combination of a $\mathrm{sp}^{2}$-carbon nanodomain and edge-terminating functional groups of GQDs offers facile routes to surface passivation and functionalization as well as tunable band-gap properties. Due to their high surface-to-volume ratio and defect-rich chemical structure, GQDs are utilized in a broad range of applications including optoelectronics, electro- and photocatalysis, and biological therapeutics.

A number of methods have been employed to produce GQDs, including chemical oxidation, ${ }^{1-3}$ electrochemical preparation, ${ }^{4}$ hydrothermal cutting, ${ }^{5}$ and electric arc method. ${ }^{6}$ Despite the variety of synthetic methods, top-down synthesized GQDs contain large $\mathrm{sp}^{2}$-hybridized carbon planes with some defects such as holes or heteroatoms. The optical band gap of GQDs is determined by (i) the dimension of GQDs and (ii) surface states of GQDs. The surface states of GQDs can be modified by introducing heteroatoms at defects or attaching surface functional groups. ${ }^{7-10} \mathrm{GQDs}$ are a promising alternative for a variety of applications such as bioimaging, ${ }^{11}$ photodynamic therapy, ${ }^{12}$ wound disinfection, ${ }^{13}$ heavy metal sensing, ${ }^{14}$ electrocatalysis,${ }^{15}$ and photovoltaic devices. ${ }^{16}$ In contrast to other nanomaterials, GQDs are significantly more biocompatible than other types of carbon nanomaterials such as carbon nanotubes, graphene, and graphene oxide. ${ }^{17,18}$

Nitrogen is one of the most studied elements as a dopant for the host carbon nanomaterial as the introduction of nitrogen atoms perturbs electrostatic charge of adjacent carbon atoms.

Department of Chemistry, University of Kentucky, Lexington, KY 40506, USA. E-mail: dooyoung.kim@uky.edu; Tel: +1-859-257-5597

$\dagger$ Electronic supplementary information (ESI) available. See DOI: $10.1039 / \mathrm{c} 7 \mathrm{ra0} 9252 \mathrm{k}$
Recent literature demonstrates the red-shifted emission of nitrogen-containing GQDs which is attributed to the reduction of the band gap $\mathrm{p}^{7,8}$ Additionally, the incorporation of nitrogen atoms into a $\mathrm{sp}^{2}$ carbon framework in the bottom-up synthesis of GQDs has also shown the increase of photoluminescence quantum yield (PLQY). Interestingly, these dopants have also been reported to induce the blue shift of emission due to the strong electron affinity of nitrogen atoms which invokes a partial positive charge on adjacent carbon atoms. ${ }^{19,20}$ Aside from optical properties, doping nitrogen heteroatoms into carbon nanomaterials shows a great enhancement of the electrocatalytic activity toward the oxygen reduction reaction (ORR). ${ }^{19}$

Recently, Tetsuka et al. reported that absorption/emission properties of amino-functionalized GQDs were finely tuned by controlling hydrothermal temperature from $70{ }^{\circ} \mathrm{C}$ to $150{ }^{\circ} \mathrm{C} .7$ In this report, the shift of spectral position and the enhancement of fluorescence intensity were mostly attributed to N-related surface functionalities such as primary amines and amides. However, the role of edge-terminating nitrogen sites, i.e.: pyridines and pyrroles, and core $\mathrm{N}$ sites such as quaternary $\mathrm{N}$ were largely neglected. The maximum content of nitrogen atoms was observed at the lowest hydrothermal temperature and the content of nitrogen became reduced as the temperature was raised. ${ }^{7}$ In support of these experimental observations, a recent computational work likewise shows the quantity of amine $\mathrm{N}$ is connected to the red-shifted behavior of GQDs. ${ }^{8}$ In contrast to the Tetsuka study, a recent article brought about the importance of pyridinic, pyrrolic and quaternary $\mathrm{N}$ sites. This study employed much higher hydrothermal temperatures $\left(>150^{\circ} \mathrm{C}\right)$ and observed blue-shifted emission with higher quantum yield $(\Phi=34.5 \%){ }^{9}$

Until now, there were very few studies differentiating the effect of different $\mathrm{N}$ chemical states (i.e., amines, pyridines, 
pyrroles) on the optical properties of top-down-synthesized GQDs. However, a recent work by $\mathrm{Qu}$ et al. has shown a pronounced differentiation of pyrrolic and graphitic $\mathrm{N}$ states in the bottom-up synthesized carbon nanodots. ${ }^{21}$ This report utilizes the hydrothermal dehydration of urea and citric acid to show a direct correlation between the quantity of graphitic $\mathrm{N}$ and increase of PLQY. In addition, varying the nitrogen source from urea to ethylenediamine greatly enhanced the PLQY to $94 \%$. Carbon nanodots made in this way have also been employed as fluorescent probes for in vitro and in vivo studies with excellent cellular uptake and biocompatibility in mice. ${ }^{22}$

In the present study, hydrothermal temperature was controlled as an effective method to incorporate different chemical states of nitrogen atoms into GQDs. Four different chemical states of nitrogen atoms (amine, pyridinic, pyrrolic, and quaternary) were identified and related to absorption/ emission properties. Moreover, pH-dependent emission spectra was studied to probe chemical states of nitrogen atoms and to investigate the relation between nitrogen location and emission.

The synthesis of oxidized GQDs (ox-GQDs) and the subsequent nitrogen incorporation (N-GQDs) are described in Fig. 1a. ox-GQDs were prepared by reacting $100 \mathrm{mg}$ of carbon nanoonions with a $1: 3$ ratio of concentrated $\mathrm{H}_{2} \mathrm{SO}_{4}: \mathrm{HNO}_{3}$ under $95{ }^{\circ} \mathrm{C}$ reflux and vigorous stirring for 4 hours. The reaction was terminated when the reflux solution became clear and brownish in color. Afterwards, this solution was first centrifuged at $4000 \mathrm{rpm}$ for 90 minutes to allow the precipitation of unreacted carbon nano-onions. Then, the supernatant was neutralized with $\mathrm{K}_{2} \mathrm{CO}_{3}$ and dialyzed for 6 days with a dialysis bag ( $1 \mathrm{kDa}$ MWCO) to remove excess ions from the neutralization. After

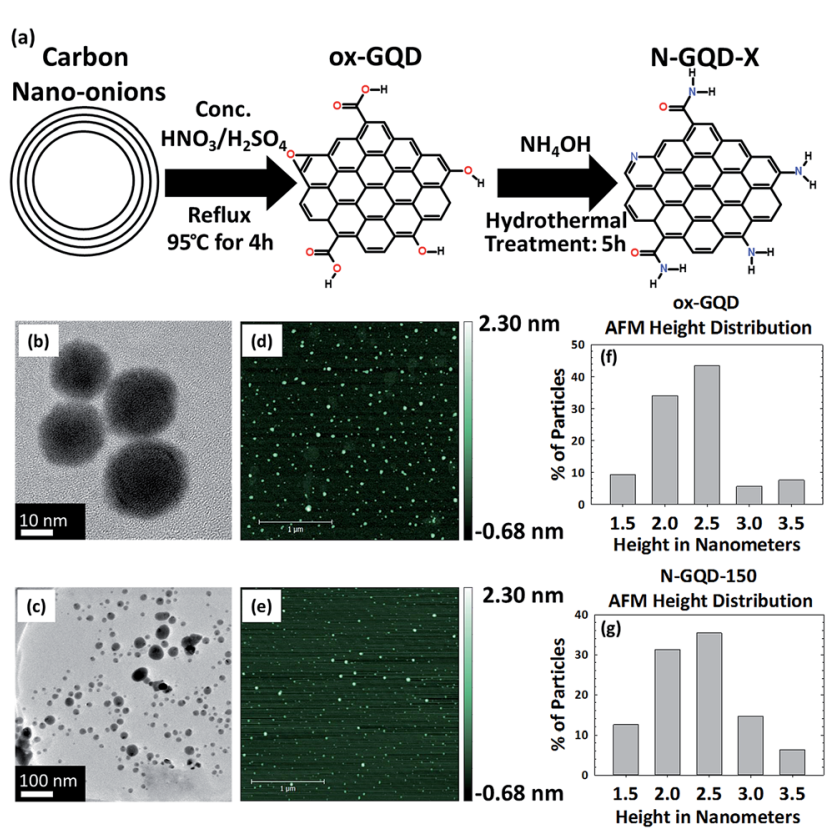

Fig. 1 (a) A scheme for the synthesis of GQDs and N-GQDs. TEM images of ox-GQD (b) and N-GQD (c), AFM images of ox-GQD (d) and $N-G Q D(e)$, and the histogram of the thickness of ox-GQDs (f) and NGQDs (g). dialysis, the resulting solution contains the purified ox-GQD in water. N-GQDs were prepared by reacting ox-GQDs with $\mathrm{NH}_{4} \mathrm{OH}$ in a hydrothermal reactor, as similar to the report by Tetsuka et al. ${ }^{7}$ First, ox-GQDs were mixed with $5.0 \mathrm{M} \mathrm{NH}_{4} \mathrm{OH}(1: 2 \mathrm{v} / \mathrm{v})$. The mixture was then placed into a $200 \mathrm{~mL}$ stainless steel autoclave reactor for 5 hours. The hydrothermal temperature of the reaction was varied from $90-190{ }^{\circ} \mathrm{C}$. N-GQD-X prepared at a specific temperature such as $150{ }^{\circ} \mathrm{C}$ will now be referred as $\mathrm{N}$ GQD-150.

Morphology and chemical structure are important factors to determine the optical behaviour of ox-GQDs and N-GQDs. The size and the thickness of ox-GQDs and N-GQDs were characterized by TEM (JEOL JEM-2200FS, $200 \mathrm{kV}$ ) and atomic force microscopic (AFM) characterizations (Park Systems XE-70). The results of TEM and AFM characterizations are shown in Fig. 1b$\mathrm{g}$. The size of ox-GQDs varies from 20-30 $\mathrm{nm}$. As the functional groups are modified, electrostatic interactions and hydrogen bonding may induce some amount of aggregation which can be seen for N-GQDs. AFM characterization of ox-GQDs and NGQDs revealed isolated particles. As shown in the histogram (Fig. 1f and g), both ox-GQD and N-GQD show an average thickness of $2.5 \mathrm{~nm}$, indicating that both particles are made of multiple stacks of graphene nanosheets. ${ }^{24}$

Hydrothermal temperature $\left(90-190{ }^{\circ} \mathrm{C}\right)$ greatly influenced the chemical functionalities and the incorporated nitrogen atoms in N-GQDs prepared. The chemical structure of N-GQDs and chemical states of nitrogen incorporated were probed by FT-IR and XPS analyses. FT-IR spectra were recorded with a Thermo Scientific Model Nicolet 6700 FT-IR Spectrometer and are displayed in Fig. S1. $\dagger$ As shown in Fig. S1, $\dagger$ FT-IR spectra of ox-GQD exhibited the presence of hydroxyl, carbonyl and carboxyl groups located at $1000 \mathrm{~cm}^{-1}(\mathrm{C}-\mathrm{O}), 1700 \mathrm{~cm}^{-1}(\mathrm{C}=\mathrm{O})$, $2500 \mathrm{~cm}^{-1}\left(\mathrm{CO}_{2} \mathrm{H}\right)$ and $3300 \mathrm{~cm}^{-1}(-\mathrm{OH})$, respectively. Compared to ox-GQDs, a new peak appeared at $1070 \mathrm{~cm}^{-1}$ in NGQD-150, which is assigned to $\mathrm{C}-\mathrm{N}$ bond. In addition, the peak at $1700 \mathrm{~cm}^{-1}$ became broadened due to the overlay of amide and carbonyl $\mathrm{C}=\mathrm{O}$. The carboxylic acid peak at $2500 \mathrm{~cm}^{-1}$ was suppressed in N-GQD-150, supporting the conversion of carboxylic group into amine or amide. Most notably, the broad peak at $3300 \mathrm{~cm}^{-1}$ in ox-GQD due to hydrogen bonded $\mathrm{O}-\mathrm{H}$ stretching became much narrower in N-GQD, indicating the substitution of hydroxyls to primary and secondary amines.

XPS characterization was conducted with a Thermo Scientific Model K-Alpha XPS instrument. The high resolution spectra were deconvoluted using Avantage software by Thermo Scientific. Peak position for each chemical state is detailed in the main text as well as in Table S1. $\dagger$ XPS analyses were conducted after drying of GQD solution mounted on a silicon wafer. Fig. 2a shows hypothetical structure of N-GQDs where nitrogen atoms are incorporated into various sites. The nitrogen-incorporated sites include pyridinic $\mathrm{N}(398.5 \mathrm{eV}, \mathrm{N} 1)$, amine $\mathrm{N}(399.7 \mathrm{eV}$, $\mathrm{N} 2$ ), pyrrolic $\mathrm{N}(400.2 \mathrm{eV}, \mathrm{N} 3)$, and quaternary $\mathrm{N}(401.3 \mathrm{eV}$, N4). N 1s peaks were assigned according to the NIST Standard Reference Database. ${ }^{27}$ The assignments of XPS peaks to different chemical states by previous studies and this report are summarized in the ESI (Table S1 $\dagger$ ). 

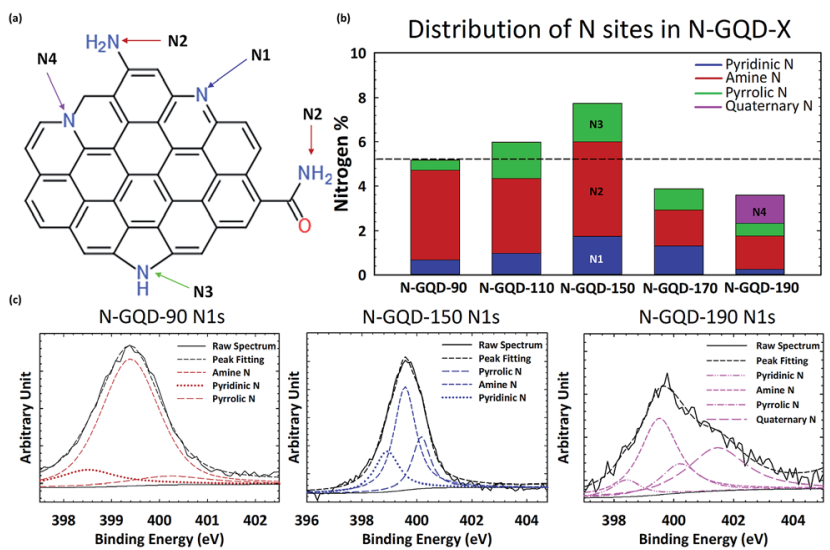

Fig. 2 (a) A simplified representation of nitrogen-incorporated GQDs. The four chemical states of nitrogen are listed as N1 (pyridinic), N2, (amine/amide), N3 (pyrrolic) and N4 (quaternary or graphitic). (b) The percentage of $\mathrm{N}$ chemical states in N-GQDs determined by N1s high resolution XPS spectra. The average nitrogen content $(5.3 \pm 1.7 \%)$ is denoted by the dashed line. (c) High resolution XPS N1s spectra of NGQD-90, N-GQD-150 and N-GQD-190.

Fig. 2c shows the high resolution XPS N1s peaks of N-GQDs synthesized at N-GQD-90, N-GQD-150, and N-GQD-190. The high resolution XPS N1s peaks of N-GQDs were deconvoluted to resolve the relative fraction of $\mathrm{N} 1, \mathrm{~N} 2, \mathrm{~N} 3$ and N4. While N-GQD90 and N-GQD-150 are mainly deconvoluted with N1, N2, and N3, the N-GQD-190 showed the significant fraction of N4 (quaternary $\mathrm{N}$ ). Fig. $2 \mathrm{~b}$ shows the evolution of the relative fraction of N1-N4 sites present in N-GQDs synthesized at different hydrothermal temperature. Clearly, the total $\mathrm{N}$ content of N-GQDs increased from $5 \%$ to $8 \%$ when hydrothermal temperature was raised up to $150{ }^{\circ} \mathrm{C}$. Then the content of $\mathrm{N}$ went down at $170{ }^{\circ} \mathrm{C}$ and $190{ }^{\circ} \mathrm{C}$. The reduction of nitrogen content for N-GQD-170 and N-GQD-190 is likely due to the hydrothermal cutting of ox-GQD, as consistent with the results reported by Luo et al. ${ }^{25}$ Overall, the content of amine groups (N2) tends to go down as temperature becomes higher. On the other hand, the content of other types of $\mathrm{N}$ edge sites such as pyridinic (N1) and pyrrolic (N3) rises until the temperature reaches $150{ }^{\circ} \mathrm{C}$. At temperatures above $150{ }^{\circ} \mathrm{C}$, the relative fraction of pyridinic $\mathrm{N}$ and pyrrolic $\mathrm{N}$ decrease. These $\mathrm{N}$ sites are converted to quaternary $\mathrm{N}$ in N-GQD-190.

Optical properties of GQDs and N-GQDs are strongly influenced by the functional groups found in the edge structure. Absorption spectra were recorded with a Thermo Scientific Evolution 201 Spectrophotometer and Emission spectra were recorded with a Jobin-Yvon Spectromax 4 Spectrofluorometer. The excitation-dependent PL spectra for ox-GQD, N-GQD-90, NGQD-150, and N-GQD-190 are shown in Fig. S2. $\dagger$ Fig. 3a shows the photograph of GQDs and N-GQDs dispersed in water under UV lamp $(\lambda=365 \mathrm{~nm}$ ). While N-GQD-90 and N-GQD-150 show green to yellow emission, N-GQD-170 and N-GQD-190 exhibited much stronger blue emission.

Fig. 3b presents UV-VIS absorption spectra of ox-GQDs and N-GQDs. The UV-Vis absorption spectra display three bands $(300 \mathrm{~nm}, 370 \mathrm{~nm}$, and $470 \mathrm{~nm})$. The $300 \mathrm{~nm}$ peak of ox-GQDs is
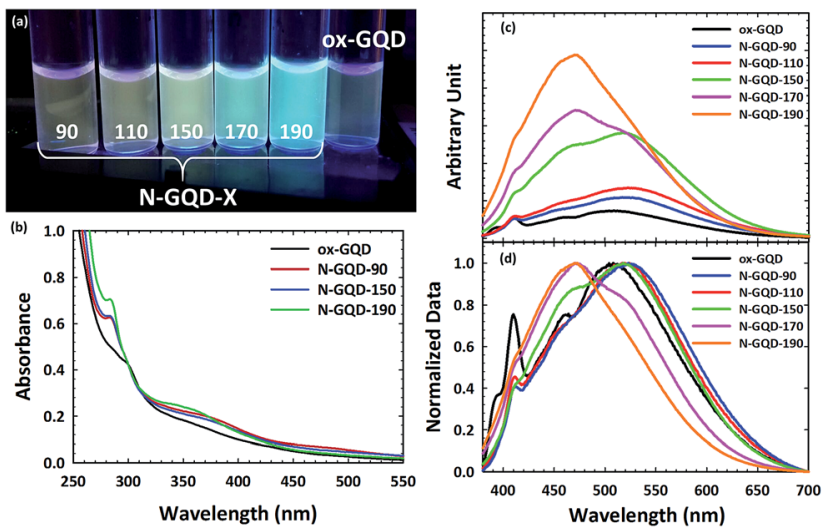

Fig. 3 (a) The photograph of ox-GQDs and N-GQDs under a UV lamp (364 nm exc) (from left: N-GQD-90, N-GQD-110, N-GQD-150, NGQD-170, N-GQD-190, and ox-GQD). (b) UV-visible absorbance, (c) emission spectra, and (d) normalized emission spectra of ox-GQDs and N-GQD-90, N-GQD-110, N-GQD-150, N-GQD-170, and NGQD-190. Emission spectra of GQDs were taken at $\lambda_{\mathrm{ex}}=360 \mathrm{~nm}$.

assigned to the $\pi-\pi^{*}$ transition and it is shifted to $280 \mathrm{~nm}$ in NGQDs. This blue-shift is attributed to the strong electron affinity of nitrogen atoms, which were reported to enhance the delocalization of electrons in the graphene nanodomain., ${ }^{\mathbf{9 1 0}}$ The peak at $370 \mathrm{~nm}$ is prominent for all N-GQDs and it is assigned to the $\mathrm{n}-\pi^{*}$ transition. This $370 \mathrm{~nm}$ band is shifted to shorter wavelength $(350 \mathrm{~nm})$ upon the increase of hydrothermal treatment temperature. This particular band is attributed to the effect of non-bonding orbitals from pyridinic $\mathrm{N}$ and pyrrolic $\mathrm{N}$ as previously described by Li et al. ${ }^{10}$ Lastly, the absorbance band at $470 \mathrm{~nm}$ that relates to another surface state $n-\pi *$ transition tends to become weaker as the hydrothermal temperature gets higher. The $470 \mathrm{~nm}$ band is likely due to the amine $\mathrm{N}$ and shows the maximum absorbance in N-GQD-90, as shown in a previous report. ${ }^{7}$ From these results, the different surface states associated with pyridinic $\mathrm{N}$, pyrrolic $\mathrm{N}$, and amine $\mathrm{N}$ show their individual impact on the absorption profile of the NGQD. It should also be noted that hydrothermal temperaturedependent evolutions of UV-VIS spectra are consistent with nitrogen-related chemical states revealed in the XPS analysis discussed above.

Fig. 3c shows the emission spectra of ox-GQD and N-GQDs. Emission spectra were taken at excitation wavelength of $360 \mathrm{~nm}$. As can be seen, emission intensity gradually increases as the hydrothermal temperature was raised. There are two major bands observed in the emission spectra of N-GQDs: $470 \mathrm{~nm}$ and $525 \mathrm{~nm}$. Compared to ox-GQD, N-GQD-90 and NGQD-110 show the red-shift of emission from $500 \mathrm{~nm}$ to $525 \mathrm{~nm}$. The $525 \mathrm{~nm}$ emission is attributed to the surface state associated with amine $\mathrm{N}$ sites. N-GQD-150 shows the emergence of a prominent new band at $470 \mathrm{~nm}$. This band is related to the maximal content of pyrrolic N. ${ }^{10}$ For N-GQD-170, the emission at $525 \mathrm{~nm}$ is suppressed; however, the emission at $470 \mathrm{~nm}$ is greatly enhanced. Based upon the results of XPS analysis, the $470 \mathrm{~nm}$ emission comes primarily from pyrrolic $\mathrm{N}$, while the $525 \mathrm{~nm}$ emission is related to the amine $\mathrm{N}$ sites. N-GQD-190 
shows the emission enhancement at $470 \mathrm{~nm}$ is even greater and is assigned to the addition of quaternary $\mathrm{N}$. The increased emission intensity at $470 \mathrm{~nm}$ follows a similar trend to the previously mentioned work by Qu et al. ${ }^{21}$ As in that report, our results likewise show that the presence of pyrrolic $\mathrm{N}$ and quaternary N strongly contributes to the enhanced PLQY. More details on the measured PLQY can be found in the ESI. $\dagger$ Overall, the samples which were hydrothermally treated at low temperatures contain large concentrations of amine and pyridinic $\mathrm{N}$ and displayed red-shifted emission profiles. Conversely, high hydrothermal treatment temperatures drive nitrogen addition toward pyrrolic and quaternary $\mathrm{N}$ sites. These absorption and emission results clearly demonstrate the resolved energetics of four different N-related chemical states in N-GQDs.

In order to further probe surface functionalities of GQDs and N-GQDs, the emission of ox-GQD and N-GQDs was explored as a function of $\mathrm{pH}$. The effects of tuning the quantity of carboxylic acids and phenols have been studied by Mei et al. ${ }^{23}$ and Luo et $a{ }^{25}$ Both functional groups were found to promote nonradiative processes with red-shifted emission. Although $\mathrm{pH}$ dependence of GQDs and polyethylene glycol (PEG)-modified GQDs were reported by Zhu et al. ${ }^{26}$ and Jin et al., ${ }^{8} \mathrm{~N}-G Q D s$ with primary amines/amides, pyridinic, etc. toward the change of $\mathrm{pH}$ has been rarely reported. In ox-GQDs, the pH-sensitive functional groups are carboxylic acids $\left(\mathrm{p} K_{\mathrm{a}} \sim 5\right)$ and phenols $\left(\mathrm{p} K_{\mathrm{a}} \sim 10\right)$. Fig. 4 a shows the $\mathrm{pH}$ dependence of ox-GQDs shows a similar trend as previous reports. ${ }^{\mathbf{8 2 6}}$ Major $\mathrm{pH}$-induced changes observed for ox-GQDs are the enhancement of emission intensity from $\mathrm{pH}=5$ to $\mathrm{pH}=7$ and the suppression of emission intensity from $\mathrm{pH}=7$ to $\mathrm{pH}=9$. While $\mathrm{pH}$ influences the emission intensity of ox-GQD, a slight shift of emission position was also observed. The spectral position change is clearly coupled with the protonation/de-protonation of carboxylic acids and phenols around their respective $\mathrm{p} K_{\mathrm{a}}$ values. The origin may be due to either the variation of absorption crosssection in the formed charged species or non-radiative interaction with environment. The normalized emission spectra in Fig. 4b show slightly red-shifted emission maxima with increasing $\mathrm{pH}$ which can also be linked to additional nonradiative processes such as collisional quenching.

Fig. 4c and $\mathrm{d}$ show the $\mathrm{pH}$ dependent emission of N-GQD150. The most pronounced change in PL intensity is the significant emission enhancement at $520 \mathrm{~nm}$ from $\mathrm{pH} 5$ to $\mathrm{pH}$
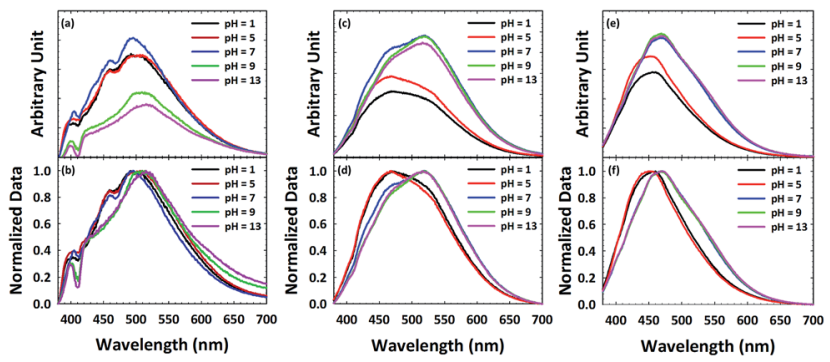

Fig. 4 Emission spectra of ( $a$ and b) Ox-GQD, (c and d) N-GQD-150 and (e and f) N-GQD-190 excited with $360 \mathrm{~nm}$ light plotted as a function of $\mathrm{pH}$.
7. Little change is observed above $\mathrm{pH}$ 7. This change of emission intensity is attributed to protonation/deprotonation of either pyridine $\mathrm{N}$ or amine $\mathrm{N}$ sites which both have a $\mathrm{p} K_{\mathrm{a}} \sim 5$. The contrast between ox-GQDs and N-GQD-150 clearly indicates the difference in surface chemistry of the two GQDs. As reported in the work by Luo, ${ }^{25}$ hydrothermal treatment removes hydroxyl groups, so little change in PL intensity above $\mathrm{pH} 7$ is expected. The different behaviour of the emission peaks at $460 \mathrm{~nm}$ and $520 \mathrm{~nm}$ indicates that the two peaks have different origins. The normalized PL spectra of N-GQD-150 (Fig. 4d) show the red-shift of emission as the $\mathrm{pH}$ changes from 5 to 7.

The PL spectra of N-GQD-190 (Fig. 4e and f) shows the $\mathrm{pH}$ dependence similar to that of N-GQD-150, but to a lesser extent. This is likely due to the reduced total content of nitrogen in $\mathrm{N}$ GQD-190, along with the smallest quantity of pH-sensitive functional groups (amine and pyridine) among all N-GQD samples. The origin of the emission around $470 \mathrm{~nm}$ in $\mathrm{N}$ GQD-150 and N-GQD-190 is hypothesized as pyrrolic and quaternary nitrogen. These two functional groups have a negligible $\mathrm{pH}$ dependence in aqueous media, so the $470 \mathrm{~nm}$ emission would be unaffected by $\mathrm{pH}$ change. The enhanced emission intensity at $\mathrm{pH}>7$ is due to the contribution of $520 \mathrm{~nm}$ emission coming from amine and pyridinic $\mathrm{N}$ sites. As can be seen in Fig. 4e and f, N-GQD-190 also shows the redshifted emission maxima but to a lesser degree due to its smaller quantity of amine and pyridinic $\mathrm{N}$ sites.

The results herein clearly demonstrate the relation between nitrogen-related surface states and emission property. Overall, with the increase of the hydrothermal temperature, the amount of pyridinic and pyrrolic nitrogens relative to amine nitrogen is enhanced. The total content of nitrogen atoms was found to be maximal at $150{ }^{\circ} \mathrm{C}$. At lower temperatures, amine groups were predominant as a result of a kinetically-favored addition of aqueous ammonia. At higher temperature, surface amines are converted to edge-sites (pyridinic or pyrrolic), core $\mathrm{N}$ sites (quaternary), or partially removed signifying a thermodynamically favourable process. These results clearly differentiate the impact of $\mathrm{N}$ chemical states on optical behaviour of GQDs. This understanding is critical for developing finely tuned, highperformance nanoparticles that are also more environmentallyfriendly. Fundamentally understanding the effects that specific nitrogen chemical states have on carbon nanomaterials will bolster their potential use in optoelectronics, bioimaging, photo- and electrocatalytic applications.

\section{Conflicts of interest}

There are no conflicts to declare.

\section{Acknowledgements}

The authors thank Prof. Ambrose Seo and John Connell for AFM characterization. The authors appreciate Dali Qian for TEM characterizations. This work was supported by the National Science Foundation under Cooperative Agreement No. 1355438. The purchase of a new XPS system recently installed at the 
University of Kentucky was supported by the fund from the NSF EPSCoR grant (grant no. 0814194).

\section{Notes and references}

1 J. Peng, W. Gao, B. K. Gupta, Z. Liu, R. Romero-Aburto, L. Ge, L. Song, L. B. Alemany, X. Zhan, G. Gao, S. A. Vithayathil, B. A. Kaipparettu, A. A. Marti, T. Hayashi, J. J. Zhu and P. M. Ajayan, Nano Lett., 2012, 12, 844.

2 Y. Dong, C. Chen, X. Zheng, L. Gao, Z. Cui, H. Yang, C. Guo, Y. Chi and C. M. Li, J. Mater. Chem., 2012, 22, 8764.

3 H. Tao, K. Yang, Z. Ma, J. Wan, Y. Zhang, Z. Kang and Z. Liu, Small, 2012, 8, 281.

4 D. B. Shinde and V. K. Pillai, Chem.-Eur. J., 2012, 18, 12522. 5 D. Pan, J. Zhang, Z. Li and M. Wu, Adv. Mater., 2010, 22, 734.

6 M. Bottini, C. Balasubramanian, M. I. Dawson, A. Bergamaschi, S. Bellucci and T. J. Mustelin, J. Phys. Chem. B, 2006, 110(2), 831.

7 H. Tetsuka, R. Asahi, A. Nagoya, K. Okamoto, I. Tajima, R. Ohta and A. Okamoto, Adv. Mater., 2012, 24, 5333.

8 S. H. Jin, D. H. Kim, G. W. Jun, S. H. Hong and S. Jeon, ACS Nano, 2013, 7(2), 1239.

9 Y. Dai, H. Long, X. Wang, Y. Wang, Q. Gu, W. Jiang, Y. Wang, C. Li, T. H. Zeng, Y. Sun and J. Zeng, Part. Part. Syst. Charact., 2014, 31, 597.

10 Y. Li, Y. Zhao, H. Cheng, Y. Hu, G. Shi, L. Dai and L. Qu, J. Am. Chem. Soc., 2012, 134, 15.

11 S. Zhu, J. Zhang, C. Qiao, S. Tang, Y. Li, W. Yuan, B. Li, L. Tian, F. Liu, R. Hu, H. Gao, H. Wei, H. Zhang, H. Sun and B. Yang, Chem. Commun., 2011, 47, 6858.

12 Z. M. Markovic, B. Z. Ristic, K. M. Arsikin, D. G. Klisic, L. M. Harhaji-Trajkovic, B. M. Todorovic-Markovic, D. P. Kepic, T. K. Kravic-Stevovic, S. P. Jovanovic,
M. M. Milenkovic, D. D. Milivojevic, V. Z. Bumbasirevic, M. D. Dramicanin and V. S. Trajkovic, Biomaterials, 2012, 33, 7084.

13 H. Sun, N. Gao, K. Dong, J. Ren and X. Qu, ACS Nano, 2014, 8(6), 6202.

14 H. Sun, N. Gao, L. Wu, J. Ren, W. Wei and X. Qu, Chem.-Eur. J., 2013, 19, 13362.

15 Q. Li, S. Zhang, L. Dai and L. Li, J. Am. Chem. Soc., 2012, 134(46), 18932.

16 Y. Li, Y. Hu, Y. Zhao, G. Shi, L. Deng, Y. Hou and L. Qu, Adv. Mater., 2011, 23, 776.

17 X. Zhang, Y. Zhu, J. Li, Z. Zhu, J. Li, W. Li and Q. Huang, J. Nanopart. Res., 2011, 13, 6941.

18 C. Wu, C. Wang, T. Han, X. Zhou, S. Guo and J. Zhang, Adv. Healthcare Mater., 2013, 2, 1613.

19 K. Gong, F. Du, Z. Xia, M. Durstock and L. Dai, Science, 2009, 323(5915), 760.

20 S. Y. Wang, D. S. Yu and L. M. Dai, J. Am. Chem. Soc., 2011, 133, 5182.

21 D. Qu, M. Zheng, L. Zhang, H. Zhao, Z. Xie, X. Jing, R. E. Haddad, H. Fan and Z. Sun, Sci. Rep., 2014, 4, 5294.

22 M. Zheng, S. Liu, J. Li, Z. Xie, D. Qu, X. Miao, X. Jing, Z. Sun and H. Fan, J. Mater. Res., 2015, 30, 3386.

23 Q. Mei and Z. Zhang, Angew. Chem., Int. Ed., 2012, 51, 5602. 24 D. Pan, J. Zhang, Z. Li and M. Wu, Adv. Mater., 2010, 22, 734.

25 P. Luo, Y. Qiu, X. Guan and L. Jiang, Phys. Chem. Chem. Phys., 2014, 16, 19011.

26 S. Zhu, J. Zhang, X. Liu, B. Li, X. Wang, S. Tang, Q. Meng, Y. Li, C. Shi, R. Hu and B. Yang, RSC Adv., 2012, 2, 2717.

27 A. V. Naumkin, A. Kraut-Vass, S. W. Gaarenstroom and C. J. Powell, NIST Standard Reference Database 20, Version 4.1, 2012. 